\title{
miR-18a-5p promotes cell invasion and migration of osteosarcoma by directly targeting IRF2
}

\author{
CHAO LU ${ }^{1}$, KAN PENG $^{1}$, HAO GUO $^{1}$, XIAOYU REN $^{1}$, SHOUYE HU $^{1}$, \\ YUANZHEN CAI ${ }^{1}$, YAN HAN ${ }^{1}$, LE MA $^{2}$ and PENG XU ${ }^{1}$ \\ ${ }^{1}$ Department of Joint Surgery, Xi'an Honghui Hospital, Xi'an Jiaotong University Health Science Center, \\ Xi'an, Shaanxi 710054; ${ }^{2}$ School of Public Health, Xi'an Jiaotong University \\ Health Science Center, Xi'an, Shaanxi 710061, P.R. China \\ Received February 6, 2018; Accepted June 19, 2018
}

DOI: $10.3892 / \mathrm{ol} .2018 .9032$

\begin{abstract}
An increasing number of studies have suggested that microRNAs (miRNAs) are involved in the progress of many human cancers including osteosarcoma (OS). Especially, microRNA-18a-5p (miR-18a-5p) has been reported to associate with the occurrence, development and clinical outcomes of human cancers. Therefore, we investigated the functions of miR-18a-5p in OS. Reverse transcription-quantitative PCR (RT-qPCR) showed that miR-18a-5p was significantly upregulated in OS tissues and cell lines (MG-63 and Saos-2). The overexpression of miR-18a-5p was found to significantly promote cell migration and invasion in MG-63 cells via Transwell assay. Moreover, luciferase reporter assays indicated that interferon regulatory factor (IRF) 2 was a direct target of miR-18a-5p. IRF2 was downregulated in MG-63 and Saos-2 cell lines. Furthermore, Transwell analysis showed that the knockout of IRF2 promoted cell migration and invasion in MG-63 cells. Carcinogenesis of miR-18a-5p was reversed by the overexpression of IRF2 in OS. In conclusion, miR-18a-5p promoted the invasion and migration of OS cells through inhibiting IRF2 expression. Thus, miR-18a-5p might act as a potential target for the diagnosis and treatment of OS in the future.
\end{abstract}

Correspondence to: Dr Le Ma, School of Public Health, Xi'an Jiaotong University Health Science Center, 76 Yanta West Road, Xi'an, Shaanxi 710061, P.R. China

E-mail: dvb577715@163.com

Dr Peng Xu, Department of Joint Surgery, Xi'an Honghui Hospital, Xi'an Jiaotong University Health Science Center, 555 Friendship Road, Xi'an, Shaanxi 710054, P.R. China

E-mail: fvx133139@163.com

Key words: osteosarcoma, miR-18a-5p, migration, invasion, interferon regulatory factor 2

\section{Introduction}

Osteosarcoma (OS) is a primary highly malignant tumor of the bone, which has a high incidence and mortality in children and adolescents aged 10-25 years (1). Moreover, OS accounts for approximately $5 \%$ of pediatric malignancies which seriously threatens the health of children (2). OS has high destructive and metastatic potential, which could occur in lung metastasis within a few months. Despite the improvement of the diagnosis and therapy, the 5-year survival rate of OS still remains poor, only $5-20 \%$ after amputation (3). Thus, it is urgent for us to further analyze and clarify the molecular mechanisms for tumorigenesis of OS.

A number of microRNAs (miRNAs) have been reported to regulate the development and malignant progression of OS functioning as oncogenes or tumor suppressors $(4,5)$. miRNAs mainly inhibit the transcription of $\mathrm{mRNA}$ or protein of relative genes by binding to the 3'-UTR of target genes (6). Especially, miR-18a was found to regulate various human cancers, such as gastric cancer (7), nasopharyngeal carcinoma (8), colorectal cancer (9) and glioblastoma (10). It indicated that miR-18a might be involved in the occurrence and development of human cancers. Moreover, the function of miR-18a was different in different cancers. For instance, miR-18a was reported to suppress cell proliferation in bladder cancer (11). However, Song et al reported that miR-18a promoted proliferation, migration and invasion of human glioblastoma cells by targeting neogenin (10). The contradictory functions of miR-18a were of interest to us as well as the molecular mechanisms of miR-18a in OS.

As a member of interferon regulatory factor (IRF) family, IRF2 has been found to participate in tumorigenesis of human cancers through modulating cell physiological activity (12). Moreover, IRF2 has been reported to act as a functional regulator in the progression of human cancers $(13,14)$. Abnormal expression of IRF2 also has been demonstrated to associate with cell invasion and tumor growth (15). In addition, overexpression of IRF2 was found to inhibit proliferation (16) and promote apoptosis (17) in hepatocellular carcinoma. Besides, the specific function of IRF2 was also identified in pancreatic and breast cancer through regulating cell physiological activity (18). However, the exact molecular mechanisms of IRF2 still remain unclear in OS and need to be further investigated. 
In the present study, the alteration of the microRNA-18a-5p (miR-18a-5p) expression was investigated in OS for the first time. Simultaneously, we explored the effect of miR-18a-5p on the migration and invasion of OS cells. The relationship between miR-18a-5p and IRF2 was analyzed as well. We aimed to provide novel ways for the diagnosis and treatment of OS.

\section{Materials and methods}

Cell culture. The MG-63, Saos-2 OS cell lines and human fetal osteoblastic cell line hFOB1.19 were acquired from the American Type Culture Collection (ATCC, Manassas, VA, USA). Then these cells were cultured in DMEM (Dulbecco's modified Eagle's medium) added with $10 \%$ fetal bovine serum (FBS). The cells were incubated in a humidified cell incubator at $37^{\circ} \mathrm{C}$ with $5 \% \mathrm{CO}_{2}$.

Tissue samples. Forty-three human OS samples were obtained from the Department of Joint Surgery, Xi'an Honghui Hospital, Xi'an Jiaotong University Health Science Center (Xi'an, China). Signed informed consents were obtained from the patients or guardians. Then the samples were quickly frozen in liquid nitrogen and stored at $-80^{\circ} \mathrm{C}$ for further experiments. This study was approved by the Research Ethics Committee of the Department of Joint Surgery, Xi'an Honghui Hospital, Xi'an Jiaotong University Health Science Center.

Transfection. The miR-18a mimics and inhibitors or miR-NC were purchased from Guangzhou RiboBio Co., Ltd. (Guangzhou, China) with the sequences as follows: mimic-sense strand, 5'-UAAGGUGCAUCUAGUGCAGA UAG-3'; mimic-antisense strand, 5'-CUAUCUGCACUAGAU GCACCUUA-3'; inhibitor strand, 5'-CUAUCUGCACUAGAU GCACCUUA-3'; and the NC is a scrambled oligonucleotide: 5'-UUCUCCGAACGUGUCACGUTT-3'. Then miR-18a-5p mimics, miR-18a-5p inhibitor or IRF2 siRNA (si-IRF2) were briefly transfected into MG-63 cells using Invitrogen ${ }^{\mathrm{TM}}$ Lipofectamine 2000 (Invitrogen: Thermo Fisher Scientific, Inc., Waltham, MA, USA). After 24-48 h, these cells were applied to the following experiments.

RNA extraction and reverse transcription-quantitative $P C R(R T-q P C R)$. TRIzol reagent (Invitrogen: Thermo Fisher Scientific, Inc.) was applied to extract total RNA, containing miRNA from OS tissue and cell lines. To obtain the cDNA templates, $1 \mu \mathrm{g}$ total RNA of each sample was used for reverse transcription using a miScript Reverse Transcription kit (Qiagen $\mathrm{GmbH}$; Hilden, Germany). This reaction was performed at $37^{\circ} \mathrm{C}$ for $60 \mathrm{~min}$, then $95^{\circ} \mathrm{C}$ for $5 \mathrm{~min}$. Quantitative PCR was conducted through the SYBR-Green assay (Invitrogen: Thermo Fisher Scientific, Inc.) using the ABI PRISM 7500 DNA Sequence Detection System (Applied Biosystems: Thermo Fisher Scientific, Inc.). U6 or GAPDH was used as a control of miR-18a-5p or IRF2. The miR-18a-5p and IRF2 expression levels were analyzed using the $2^{-\Delta \Delta \mathrm{Cq}}$ method (19).

Cell migration and invasion assay. Transwell chamber (Costar $^{\mathrm{TM}}$; Corning, Inc., Corning, NY, USA) was used to measure cell migration and invasion. In migration test,
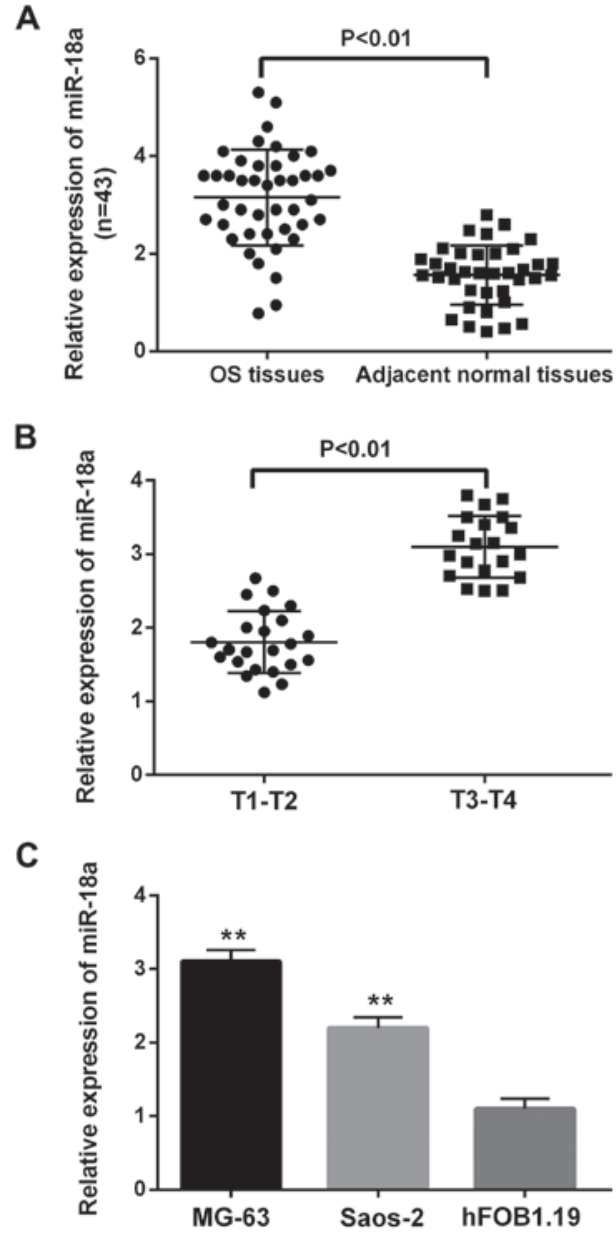

Figure 1. miR-18a-5p expression was increased in osteosarcoma tissues and cell lines. (A) The miR-18a-5p expression was upregulated in osteosarcoma tissues. (B) The expression of miR-18a-5p was related to the tumor pathological stage of osteosarcoma. (C) The miR-18a-5p expression was increased in MG-63 and Saos-2 cells compared with normal osteosarcoma cells (hFOB1.19). ${ }^{* *} \mathrm{P}<0.01$. miR-18a-5p, microRNA-18a-5p.

$5 \times 10^{4}$ cells were placed in the upper chamber with non-coated membrane to detect the ability of cell migration. Then the upper surface with Matrigel (BD Biosciences, Franklin Lakes, NJ, USA) was used to measure the ability of cell invasion. The lower chamber was filled with $10 \%$ FBS. The cells were incubated for $48 \mathrm{~h}$. Finally, the cells were stained with crystal violet staining solution (Beyotime Institute of Biotechnology, Nantong, China).

Luciferase reporter assay. The 293T cell line obtained from ATCC was used to perform luciferase reporter assay. Then the IRF2-wild and -mut were inserted into the pGL3 promoter vector (GenScript Co., Ltd., Nanjing, China) which was transfected into $293 \mathrm{~T}$ cells using Lipofectamine 2000 (Invitrogen: Thermo Fisher Scientific, Inc.) along with miR-18-5p mimics or miR-NC. After that, cells were seeded in a 96-well plate for $24 \mathrm{~h}$, and luciferase reporter assay kit (Promega Corp., Madison, WI, USA) was applied to perform luciferase assays.

Western blotting. The protein samples were obtained using RIPA buffer. Proteins were separated through SDS-PAGE 
A

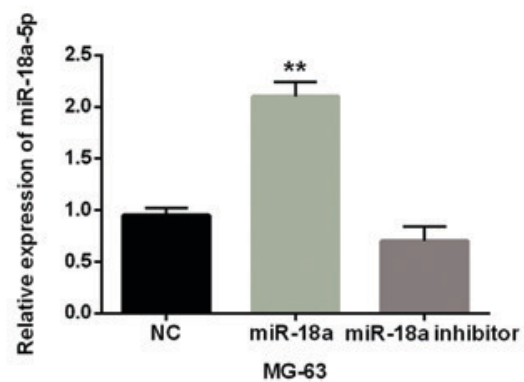

B
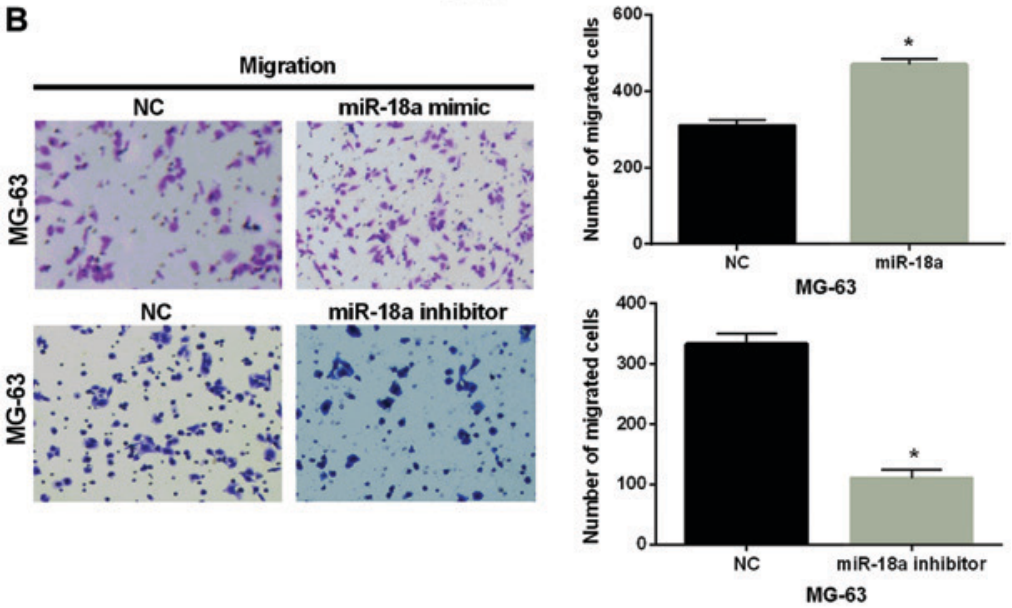

C
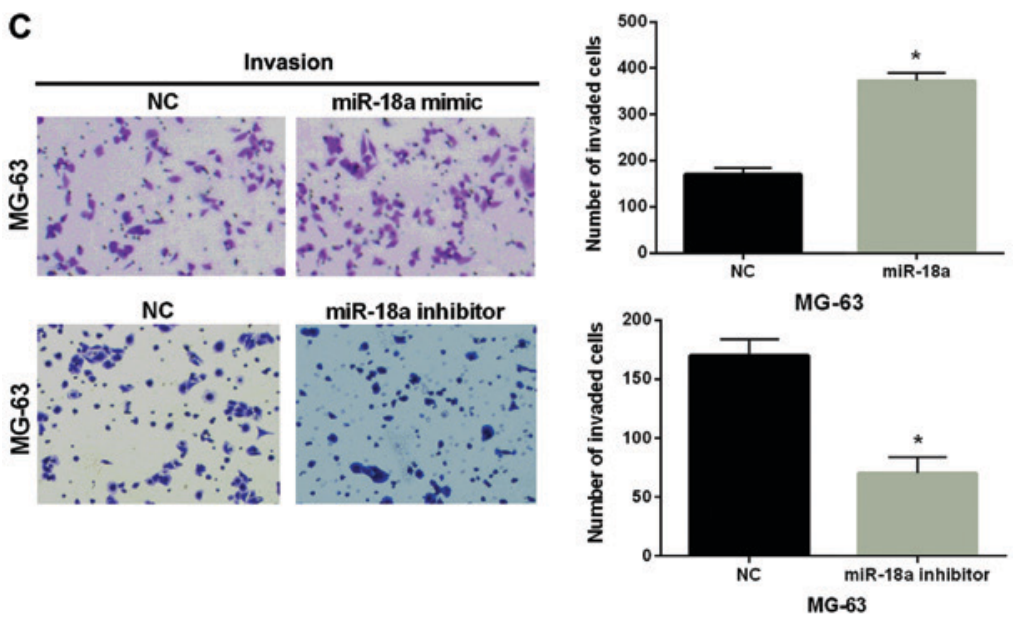

Figure 2. Overexpression of miR-18a-5p promotes cell migration and invasion in OS. (A) MG-63 cells were transfected with miR-18a-5p mimic or inhibitor, and then miR-18a-5p expression was measured via RT-qPCR. (B and C) The cell migration and invasion were detected in MG-63 cells with miR-18a-5p mimic or inhibitor. ${ }^{*} \mathrm{P}<0.05$; ${ }^{* *} \mathrm{P}<0.01$. miR-18a-5p, microRNA-18a-5p; OS, osteosarcoma; NC, normal control without miR-18a-5p mimic or inhibitor.

and incubated with $5 \%$ blocking reagent in nitrocellulose membranes at room temperature. Next we incubated the membranes overnight at $4^{\circ} \mathrm{C}$ with rabbit polyclonal anti-IRF2 antibody (dilution, 1:1,000; cat. no. ab86018; Abcam, Shanghai, China) and rabbit polyclonal anti-GAPDH antibody (dilution, 1:1,000; cat. no. ab70699; Abcam). Then, the membranes were washed 3 times and incubated with goat anti-rabbit IgG-H\&L secondary antibody (1:1,000; cat. no. ab150077; Abcam) for $1 \mathrm{~h}$ at room temperature. Protein expression levels were measured by Image Lab software (Bio-Rad Laboratories, Inc., Hercules, CA, USA).

Statistical analysis. Statistical analysis was carried out with SPSS 18.0 (SPSS, Inc., Chicago, IL, USA) and GraphPad Prism 6.0. The results are presented as mean \pm standard deviation (SD). The difference was analyzed using Student's t-test or one-way ANOVA with Tukey's post hoc test. Differences were considered significant at $\mathrm{P}<0.05$.

\section{Results}

miR-18a-5p expression is increased in OS tissues and cell lines. Firstly, the expression levels of miR-18a-5p were detected to investigate its alteration in OS. The results showed that miR-18a-5p was significantly upregulated in OS tissues compared with the adjacent normal tissues detected by RT-qPCR experiment (Fig. 1A). Moreover, we also found that the expression level of miR-18a-5p at T3-T4 stage of OS was higher than that of T1-T2 stage (Fig. 1B). It indicated that the abnormal expression of miR-18a-5p might relate to the tumor stage of OS. Similarly, upregulation of miR-18a-5p was also identified in MG-63 and Saos-2 cells compared with the normal 
A

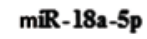

Wild of IRF 2 3'-UTR

Mutant of IRF2 3'-UTR

B
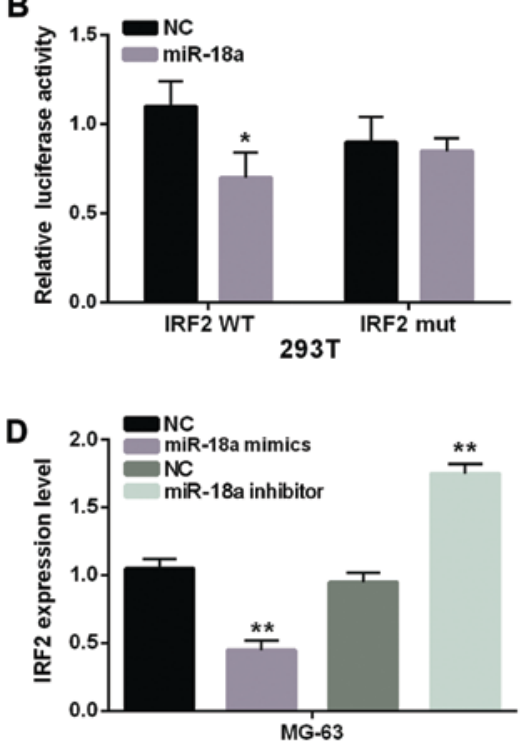

3'......GAUAGACGU GAUCUACGU GGAAU ....5'

\|\|\|\|$\|$

5'.......UUGCAGU CU GCU U CU GCACCUUA....3'

5 .......UUGCAGUCU GCUUCU CCU CGUAA.....3'

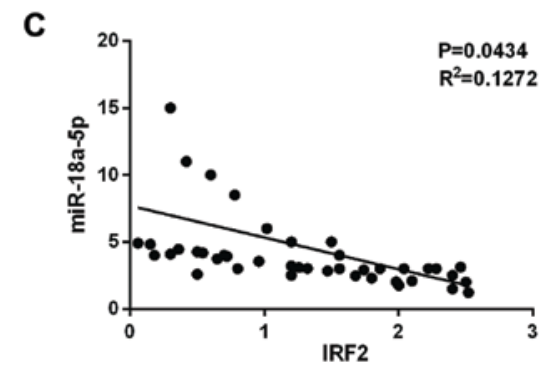

E

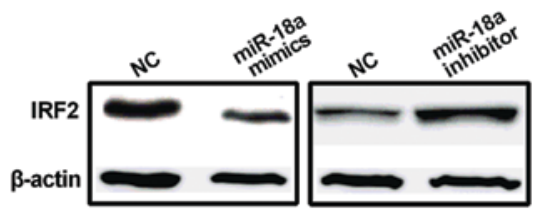

Figure 3. miR-18a-5p directly targets IRF2 in OS. (A) The binding sites of miR-18a-5p and the wild-type of IRF2. (B) Luciferase reporter assay. (C) Pearson's correlation coefficient between miR-18a-5p and IRF2 in OS tissues. (D) The mRNA expression of IRF2 was analyzed in cells transfected with miR-18a-5p mimic or inhibitor. (E) The protein expression of IRF2 was analyzed in cells transfected with miR-18a-5p mimic or inhibitor (there was a break in the gel due to the use of separate membranes). ${ }^{*} \mathrm{P}<0.05,{ }^{* *} \mathrm{P}<0.01$. miR-18a-5p, microRNA-18a-5p; IRF2, interferon regulatory factor 2; OS, osteosarcoma; NC, normal control without miR-18a-5p mimic or inhibitor.

human osteoblast hFOB1.19 cells (Fig. 1C). Then MG-63 cell line was selected to perform further experiment because of its significant difference in the expression of miR-18a-5p. Taken together, high expression of miR-18a-5p was upregulated and might be involved in the pathogenesis of OS.

Cell migration and invasion are promoted by $m i R-18 a-5 p$ in $O S$. Subsequently, we explored the effect of miR-18a-5p on cell migration and invasion in OS. First, miR-18a-5p mimic or inhibitor was transfected into MG-63 cells. The transfection efficiency was verified by RT-qPCR as shown in Fig. 2A. Then Transwell assay indicated that cell migration was markedly promoted by the overexpression of miR-18a-5p and inhibited by knockdown of miR-18a-5p compared with the control group in MG-63 cells (Fig. 2B). Similarly, miR-18a-5p mimics were also found to promote cell invasion and miR-18a-5p inhibitor inhibited cell invasion (Fig. 2C). Hence, we considered that overexpression of miR-18a-5p promoted cell migration and invasion in OS.

miR-18a-5p directly targets IRF 2 . The potential target genes of miR-18a-5p were searched through the database of TargetScan (www.targetscan.org). Remarkably, miR-18a-5p was predicted to bind with 3'-UTR region of IRF2 (Fig. 3A). To confirm the above prediction, luciferase reporter assays were performed in $293 \mathrm{~T}$ cells. The results indicated that miR-18a-5p mimic decreased the firefly luciferase reporter activity of wild-type IRF2, but this inhibition of luciferase reporter activity was not found in cells with mutated-type IRF2 (Fig. 3B). Moreover, we also found a negative correlation between miR-18a-5p and
IRF2 in OS tissues $\left(\mathrm{P}=0.0434, \mathrm{R}^{2}=0.1272\right.$, Fig. 3C). In addition, the results of RT-qPCR and western blotting indicated that the mRNA and protein expression levels of IRF2 were markedly decreased in MG-63 cells with miR-18a-5p mimics and increased in MG-63 cells with miR-18a-5p inhibitor compared to the control group (Fig. 3D and E). These results indicated that IRF2 was a direct target gene of miR-18a-5p, which had negative association with IRF2 expression in OS.

Overexpression of IRF2 counteracts the promoted effect of $m i R-18 a-5 p$ in $O S$. Furthermore, we detected the mRNA expression level of IRF2 in MG-63, Saos-2 cells and human fetal osteoblastic cell line hFOB1.19. These two cell lines had significantly low expression of IRF2 compared with hFOB1.19 cell line (Fig. 4A). Then the transfection efficiency was verified in MG-63 cells with IRF2 siRNA as shown in Fig. 4B. Furthermore, the cell migration and invasion assay in MG-63 cells with IRF2 siRNA were performed to explore the role of IRF2 in OS. Moreover, Transwell assay suggested that IRF2 siRNA obviously promoted the migration and invasion of MG-63 cells (Fig. 4C and D). Besides, the IRF2 plasmid and miR-18a-5p mimics were co-transfected into MG-63 cells to further confirm the biological interrelationship of miR-18a-5p and IRF2 in OS. Then the expression of IRF2 was examined in MG-63 cells with miR-18a-5p and IRF2. We found that mRNA and protein expression levels of IRF2 were decreased by miR-18a mimics while there was little change in cells with IRF2 plasmid and miR-18a-5p mimics (Fig. 5A and B). Next, the cell migration and invasion were also analyzed in these transfected cells. Transwell assay showed that overexpression of IRF2 partially 

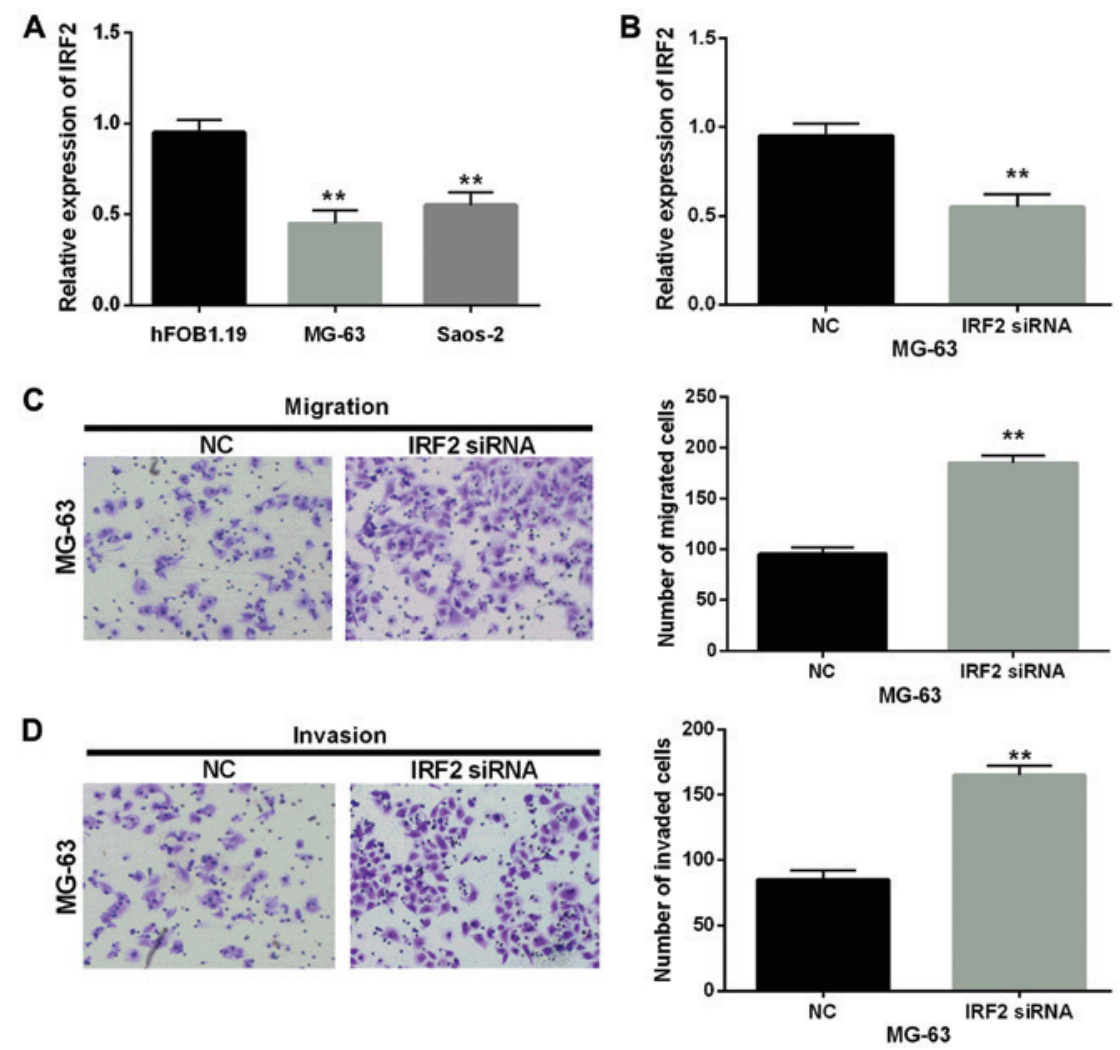

Figure 4. Knockout of IRF2 promotes cell migration and invasion in OS. (A) IRF2 expression was decreased in MG-63 and Saos-2 cells compared with normal OS cells (hFOB1.19). (B) IRF2 expression was examined through RT-qPCR in MG-63 cells transfected with si-IRF2. (C and D) Cell migration and invasion were detected in MG-63 cells with si-IRF2. ${ }^{* *} \mathrm{P}<0.01$. IRF2, interferon regulatory factor 2; OS, osteosarcoma; si-IRF2, IRF2 siRNA; NC, normal control without si-IRF2.

A

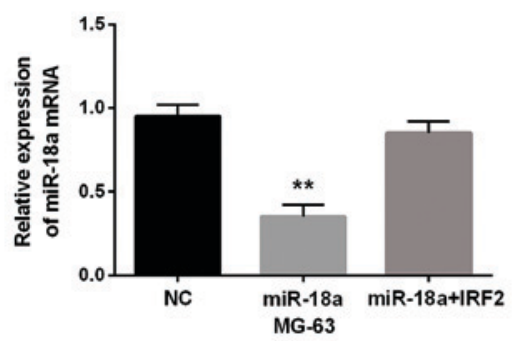

C

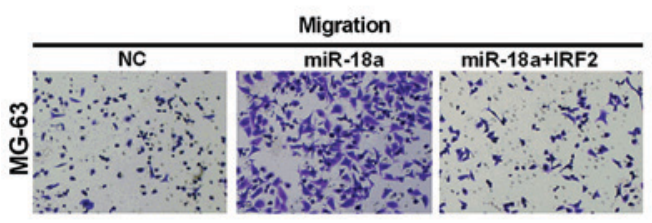

D

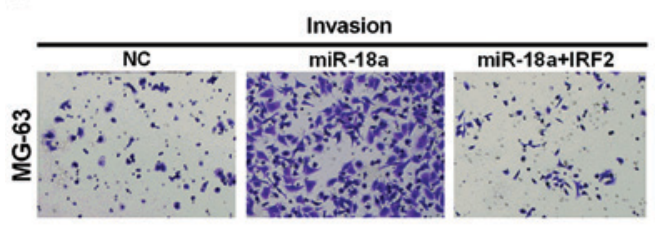

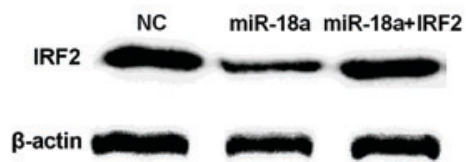
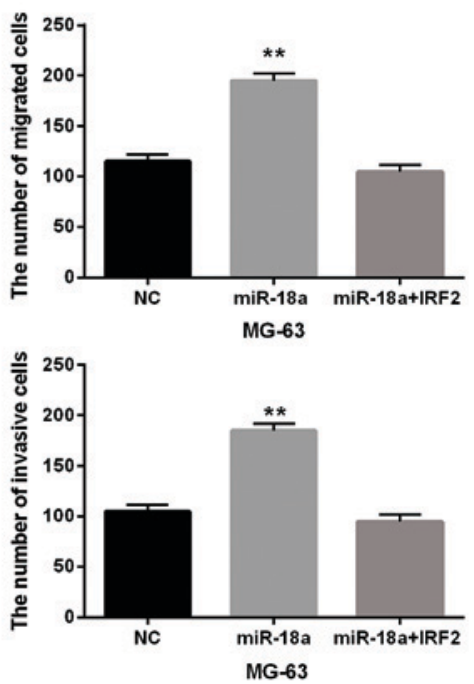

Figure 5. Overexpression of IRF2 counteracts the promoted effect of miR-18a-5p in OS. (A and B) The mRNA and protein expression of IRF2 were analyzed in cells transfected with IRF2 plasmid and miR-18a-5p mimics. (C and D) Cell migration and invasion were detected in MG-63 cells with IRF2 plasmid and miR-18a-5p mimics. ${ }^{* *} \mathrm{P}<0.01$. IRF2, interferon regulatory factor 2; miR-18a-5p, microRNA-18a-5p; OS, osteosarcoma; NC, normal control without IRF2 plasmid and miR-18a-5p mimics. 
attenuated miR-18a-mediated promotion on cell migration and invasion (Fig. 5C and D). Taken together, IRF2 acted as a tumor suppressor in OS and miR-18a-5p might promote cell invasion and migration by directly targeting IRF 2 in OS.

\section{Discussion}

Increasing number of studies proposed that miRNAs have great effect on the pathological mechanisms and development of human cancers. In this study, miR-18a-5p was found to be upregulated and to act as a tumor promoter in OS. Moreover, miR-18a-5p was identified as a member of miR-17-92 cluster including miR-17/miR-20, miR-19a/miR-19b, miR-92a and miR-18a (20), and miR-17-92 cluster was identified to be overexpressed and had an oncogenic effect in colorectal cancer, anaplastic thyroid cancer and renal cell carcinoma which was consistent with our findings on miR-18a-5p in OS (21-24). Furthermore, miR-18a-5p was found to directly target IRF2 in OS in this study. Moreover, miR-18a was reported to target IRF2 in gastric cancer (25) and lung cancer (13) which was similar to our result. In addition, Liu et al demonstrated that IRF2 suppressed the progression of lung cancer (26). We found the inhibitory effect of IRF2 on cell migration and invasion in OS.

Importantly, previous studies reported that the function of miRNAs was different in OS. Some miRNAs were found to function as oncogenes, while others acted as tumor suppressors. For instance, miR-126 was found to inhibit proliferation, migration, invasion and EMT in OS by targeting ZEB1 (27). miR-203 inhibited proliferation and promoted apoptosis of OS cells (28). Besides, miR-93 was upregulated and acted as oncogene in OS cells (29). In the present study, miR-18a-5p was also identified to be upregulated and to act as oncogene in OS cells. Moreover, we also identified that miR-18a-5p was associated with the pathological stage of OS.

As a direct target of miR-18a-5p, IRF2 is a DNA-binding protein belonging to IRFs family (30). The functions of IRF2 may be complex and remain obscure in human cancers. Previous studies demonstrated that IRF2 inhibited gene transcription induced by interferon, while other reports found that it had transcriptional activation function (14,31-33). We confirmed the role of IRF2 in OS. In this study, we found that IRF2 functioned as a tumor suppressor by inhibiting the migration and invasion of OS cells. Overexpression of IRF2 could counteract the promoted effect of miR-18a-5p in OS. Therefore, we considered that miR-18a-5p promoted cell invasion and migration in OS by directly targeting IRF2.

In conclusion, miR-18a-5p was upregulated in OS tissues and cell lines, and miR-18a-5p promoted cell migration and invasion in OS by inhibiting IRF2 expression. Ultimately, miR-18a-5p may become a potential therapeutic target in OS.

\section{Acknowledgements}

Not applicable.

\section{Funding}

This study was funded by the China Postdoctoral Science Foundation (no. 2017M613179) and the National Natural Science Foundation of China (no. 81691877).

\section{Availability of data and materials}

The datasets used and/or analyzed during the present study are available from the corresponding author on reasonable request.

\section{Authors' contributions}

CL conceived and designed the study and wrote the manuscript. KP, HG and XR performed the data analyses. SH, YC and $\mathrm{YH}$ performed the histological examination of the OS. LM and PX contributed to the conception of the study. All authors read and approved the final manuscript.

\section{Ethics approval and consent to participate}

The study was approved by the Ethics Committee of the Department of Joint Surgery, Xi'an Honghui Hospital, Xi'an Jiaotong University Health Science Center (Xi'an, China). Signed informed consents were obtained from the patients or guardians.

\section{Patient consent for publication}

Not applicable.

\section{Competing interests}

The authors declare that they have no competing interests.

\section{References}

1. Poletajew S, Fus L and Wasiutyński A: Current concepts on pathogenesis and biology of metastatic osteosarcoma tumors. Ortop Traumatol Rehabil 13: 537-545, 2011.

2. Lewis VO: What's new in musculoskeletal oncology. J Bone Joint Surg Am 89: 1399-1407, 2007.

3. Fujiwara T, Katsuda T, Hagiwara K, Kosaka N, Yoshioka Y, Takahashi RU, Takeshita F, Kubota D, Kondo T, Ichikawa H, et al: Clinical relevance and therapeutic significance of microRNA-133a expression profiles and functions in malignant osteosarcoma-initiating cells. Stem Cells 32: 959-973, 2014

4. Debebe Z and Rathmell WK: Ror2 as a therapeutic target in cancer. Pharmacol Ther 150: 143-148, 2015.

5. Geng S, Zhang X, Chen J, Liu X, Zhang H, Xu X, Ma Y, Li B, Zhang Y, Bi Z, et al: The tumor suppressor role of miR-124 in osteosarcoma. PLoS One 9: e91566, 2014.

6. Ambros V: The functions of animal microRNAs. Nature 431: 350-355, 2004.

7. LiX,Luo F,LiQ, Xu M,Feng D, Zhang G and Wu W: Identification of new aberrantly expressed miRNAs in intestinal-type gastric cancer and its clinical significance. Oncol Rep 26: 1431-1439, 2011.

8. Luo Z, Dai Y, Zhang L, Jiang C, Li Z, Yang J, McCarthy JB, She $\mathrm{X}$, Zhang $\mathrm{W}$, Ma J, et al: miR-18a promotes malignant progression by impairing microRNA biogenesis in nasopharyngeal carcinoma. Carcinogenesis 34: 415-425, 2013.

9. Wu CW, Dong YJ, Liang QY, He XQ, Ng SS, Chan FK, Sung JJ and $\mathrm{Yu}$ J: MicroRNA-18a attenuates DNA damage repair through suppressing the expression of ataxia telangiectasia mutated in colorectal cancer. PLoS One 8: e57036, 2013.

10. Song Y, Wang P, Zhao W, Yao Y, Liu X, Ma J, Xue Y and Liu Y: MiR-18a regulates the proliferation, migration and invasion of human glioblastoma cell by targeting neogenin. Exp Cell Res 324: 54-64, 2014.

11. Tao J, Wu D, Li P, Xu B, Lu Q and Zhang W: microRNA-18a, a member of the oncogenic miR-17-92 cluster, targets Dicer and suppresses cell proliferation in bladder cancer T24 cells. Mol Med Rep 5: 167-172, 2012. 
12. Yanai $H$, Negishi $H$ and Taniguchi T: The IRF family of transcription factors: Inception, impact and implications in oncogenesis. Oncoimmunology 1: 1376-1386, 2012.

13. Chen YJ, Wu H, Zhu JM, Li XD, Luo SW, Dong L, Liu TT and Shen XZ: MicroRNA-18a modulates P53 expression by targeting IRF2 in gastric cancer patients. J Gastroenterol Hepatol 31: 155-163, 2016.

14. Sakai T, Mashima H, Yamada Y, Goto T, Sato W, Dohmen T, Kamada K, Yoshioka M, Uchinami H, Yamamoto Y, et al: The roles of interferon regulatory factors 1 and 2 in the progression of human pancreatic cancer. Pancreas 43: 909-916, 2014.

15. Nicolini A, Carpi A and Rossi G: Cytokines in breast cancer. Cytokine Growth Factor Rev 17: 325-337, 2006.

16. Dorand RD, Nthale J, Myers JT, Barkauskas DS, Avril S, Chirieleison SM, Pareek TK, Abbott DW, Stearns DS, Letterio JJ, et al: Cdk5 disruption attenuates tumor PD-L1 expression and promotes antitumor immunity. Science 353: 399-403, 2016.

17. Amaddeo G, Guichard C, Imbeaud S and Zucman-Rossi J: Next-generation sequencing identified new oncogenes and tumor suppressor genes in human hepatic tumors. Oncoimmunology 1: $1612-1613,2012$.

18. $\mathrm{Xi} \mathrm{H}$ and Blanck $\mathrm{G}$ : Interferon regulatory factor- 2 point mutations in human pancreatic tumors. Int J Cancer 87: 803-808, 2000

19. Livak KJ and Schmittgen TD: Analysis of relative gene expression data using real-time quantitative PCR and the 2(-Delta Delta C(T)) method. Methods 25: 402-408, 2001

20. Hayashita Y, Osada H, Tatematsu Y, Yamada H, Yanagisawa K, Tomida S, Yatabe Y, Kawahara K, Sekido Y and Takahashi T: A polycistronic microRNA cluster, miR-17-92, is overexpressed in human lung cancers and enhances cell proliferation. Cancer Res 65: 9628-9632, 2005

21. Takakura S, Mitsutake N, Nakashima M, Namba H, Saenko VA Rogounovitch TI, Nakazawa Y, Hayashi T, Ohtsuru A and Yamashita S: Oncogenic role of miR-17-92 cluster in anaplastic thyroid cancer cells. Cancer Sci 99: 1147-1154, 2008.

22. Diosdado B, van de Wiel MA, Terhaar Sive Droste JS, Mongera S, Postma C, Meijerink WJ, Carvalho B and Meijer GA: MiR-17-92 cluster is associated with $13 \mathrm{q}$ gain and c-myc expression during colorectal adenoma to adenocarcinoma progression. $\mathrm{Br} \mathrm{J}$ Cancer 101: 707-714, 2009.

23. Chow TF, Mankaruos M, Scorilas A, Youssef Y, Girgis A, Mossad S, Metias S, Rofael Y, Honey RJ, Stewart R, et al: The miR-17-92 cluster is overexpressed in and has an oncogenic effect on renal cell carcinoma. J Urol 183: 743-751, 2010.
24. van Haaften G and Agami R: Tumorigenicity of the miR-17-92 cluster distilled. Genes Dev 24: 1-4, 2010.

25. Sarkar S, Balasuriya UB, Horohov DW and Chambers TM Equine herpesvirus-1 infection disrupts interferon regulatory factor-3 (IRF-3) signaling pathways in equine endothelial cells. Vet Immunol Immunopathol 173: 1-9, 2016.

26. Liu F, Yu X, Huang H, Chen X, Wang J, Zhang X and Lin Q: Upregulation of microRNA-450 inhibits the progression of lung cancer in vitro and in vivo by targeting interferon regulatory factor 2. Int J Mol Med 38: 283-290, 2016.

27. Liang C, Zhang X, Wang HM, Liu XM, Zhang XJ, Zheng B, Qian GR and Ma ZL: MicroRNA-18a-5p functions as an oncogene by directly targeting IRF2 in lung cancer. Cell Death Dis 8: e2764, 2017.

28. He Y and Yu B: MicroRNA-93 promotes cell proliferation by directly targeting P21 in osteosarcoma cells. Exp Ther Med 13: 2003-2011, 2017.

29. Jiang R, Zhang C, Liu G, Gu R and Wu H: MicroRNA-126 inhibits proliferation, migration, invasion and EMT in osteosarcoma by targeting ZEB1. J Cell Biochem 118: 3765-3774, 2017.

30. Lin W, Zhu X, Yang S, Chen X, Wang L, Huang Z, Ding Y, Huang L and Lv C: MicroRNA-203 inhibits proliferation and invasion, and promotes apoptosis of osteosarcoma cells by targeting Runt-related transcription factor 2. Biomed Pharmacother 91: 1075-1084, 2017.

31. Mamane Y, Heylbroeck C, Génin P, Algarté M, Servant MJ, LePage C, DeLuca C, Kwon H, Lin R and Hiscott J: Interferon regulatory factors: The next generation. Gene 237: 1-14, 1999.

32. Harada H, Fujita T, Miyamoto M, Kimura Y, Maruyama M, Furia A, Miyata T and Taniguchi T: Structurally similar but functionally distinct factors, IRF-1 and IRF-2, bind to the same regulatory elements of IFN and IFN-inducible genes. Cell 58: 729-739, 1989.

33. Choo A, Palladinetti P, Holmes T, Basu S, Shen S, Lock RB, O'Brien TA, Symonds G and Dolnikov A: siRNA targeting the IRF2 transcription factor inhibits leukaemic cell growth. Int J Oncol 33: 175-183, 2008. 